\title{
Coincidence point theorems for weak graph preserving multi-valued mapping
}

\author{
Aniruth Phon-on, Areeyuth Sama-Ae*, Nifatamah Makaje, Pakwan Riyapan and Saofee Busaman
}

\author{
"Correspondence: \\ sassaare@gmail.com \\ Department of Mathematics and \\ Computer Science, Faculty of \\ Science and Technology, Prince of \\ Songkla University, Pattani Campus, \\ Pattani, 94000, Thailand
}

\begin{abstract}
In this paper, we prove some coincidence and fixed point theorems for a new type of multi-valued weak $G$-contraction mapping with compact values. The results of this paper extend and generalize several known results from a complete metric space endowed with a graph. Some examples are given to illustrate the usability of our results.
\end{abstract}

MSC: $47 \mathrm{H} 04 ; 47 \mathrm{H} 10$

Keywords: fixed point theorem; multi-valued mapping; weak graph preserving; partially ordered set

\section{Introduction}

The classical contraction mapping principle of Banach states that if $(X, d)$ is a complete metric space and $f: X \rightarrow X$ is a contraction mapping, i.e., $d(f(x), f(y)) \leq \alpha d(x, y)$ for all $x, y \in X$, where $\alpha \in[0,1)$, then $f$ has a unique fixed point. Banach fixed point theorem plays an important role in several branches of mathematics. For instance, it has been used to study the existence of solutions of nonlinear integral equations, system of linear equations, nonlinear differential equations in Banach spaces and to prove the convergence of algorithms in computational mathematics. Because of its usefulness for mathematical theory, Banach fixed point theorem has been extended in many directions; see [1-11]. Several well-known fixed point theorems of single-valued mappings such as Banach and Schauder have been extended to multi-valued mappings in Banach spaces.

Fixed point theory of multi-valued mappings plays an important role in control theory, optimization, partial differential equations, economics, and applied science. For a metric space $(X, d)$, we let $C B(X)$ and $\operatorname{Comp}(X)$ be the set of all nonempty closed bounded subsets and the set of all nonempty compact subsets of $X$, respectively. A point $x$ in $X$ is a fixed point of a multi-valued mapping $T: X \rightarrow 2^{X}$ if $x$ is in $T x$.

Nadler [12] has proved a multi-valued version of the Banach contraction principle which states that each closed and bounded value contraction map on a complete metric space has a fixed point. One of the most general fixed point theorems for multi-valued nonexpansive self-mappings was studied by Kirk and Massa in 1990 [13]. They proved the existence of fixed points in Banach spaces for which the asymptotic center of a bounded sequence in a closed convex subset is nonempty and compact.

The following theorem is the first well-known theorem of multi-valued contractions studied by Nadler in 1969 [12].

(2014 Phon-on et al: licensee Springer. This is an Open Access article distributed under the terms of the Creative Commons Attribution License (http://creativecommons.org/licenses/by/2.0), which permits unrestricted use, distribution, and reproduction in any medium, provided the original work is properly cited. 
Theorem 1.1 Let $(X, d)$ be a complete metric space and $T: X \rightarrow C B(X)$. Assume that there exists $k \in[0,1)$ such that

$$
H(T x, T y) \leq k d(x, y)
$$

for all $x, y \in X$. Then there exists $z \in X$ such that $z \in T z$.

Reich [14] extended Nadler's fixed point theorem as follows.

Theorem 1.2 Let $(X, d)$ be a complete metric space and $T: X \rightarrow \operatorname{Comp}(X)$. Assume that there exists a function $\varphi:[0, \infty) \rightarrow[0,1)$ such that

$$
\limsup _{r \rightarrow t^{+}} \varphi(r)<1
$$

for each $t \in(0, \infty)$ and

$$
H(T x, T y) \leq \varphi(d(x, y)) d(x, y)
$$

for all $x, y \in X$. Then there exists $z \in X$ such that $z \in T z$.

The multi-valued mapping $T$ studied by Reich [14] in Theorem 1.2 has compact value, that is, $T x$ is a nonempty compact subset of $X$ for all $x$ in the spaces $X$. In 1989, Mizoguchi and Takahashi [15] relaxed the compactness assumption on the mapping to closed and bounded subsets of $X$. They proved the following theorem as a generalization of Nadler's theorem.

Theorem 1.3 Let $(X, d)$ be a complete metric space and $T: X \rightarrow C B(X)$. Assume that there exists a function $\varphi:[0, \infty) \rightarrow[0,1)$ such that

$$
\limsup _{r \rightarrow t^{+}} \varphi(r)<1
$$

for each $t \in[0, \infty)$ and

$$
H(T x, T y) \leq \varphi(d(x, y)) d(x, y)
$$

for all $x, y \in X$. Then there exists $z \in X$ such that $z \in T z$.

In 2007, Berinde and Berinde [16] gave the definition of a multi-valued weak contraction stated as follows.

Definition 1.4 Let $(X, d)$ be metric space and $T: X \rightarrow C B(X)$ a multi-valued mapping. $T$ is said to be a multi-valued weak contraction or a multi-valued $(\theta, L)$-weak contraction if there exist two constants $\theta \in(0,1)$ and $L \geq 0$ such that

$$
H(T x, T y) \leq \theta d(x, y)+L d(y, T x)
$$

for all $x, y \in X$. 
Then they extended Theorem 1.2 to the class of multi-valued weak contraction and showed that in a complete metric space, every multi-valued weak contraction has a fixed point. In the same paper, they also introduced a class of multi-valued mappings which is more general than that of weak contraction defined as follows.

Definition 1.5 Let $(X, d)$ be a metric space and $T: X \rightarrow C B(X)$ a multi-valued mapping. $T$ is said to be a generalized multi-valued $(\alpha, L)$-weak contraction if there exist a nonnegative number $L$ and a function $\alpha:[0, \infty) \rightarrow[0,1)$ satisfying $\lim \sup _{r \rightarrow t^{+}} \alpha(r)<1$ for each $t \in$ $[0, \infty)$ such that

$$
H(T x, T y) \leq \alpha(d(x, y)) d(x, y)+L d(y, T x)
$$

for all $x, y \in X$.

They showed that in a complete metric space, every generalized multi-valued $(\alpha, L)$ weak contraction has a fixed point.

In 2008, Jachymski [17] introduced the concept of 'contraction concerning a graph', called $G$-contraction and proved some fixed point results of $G$-contraction in a complete metric space endowed with a graph.

Definition 1.6 Let $(X, d)$ be a metric space and $G=(V(G), E(G))$ a directed graph such that $V(G)=X$ and $E(G)$ contains all loops, i.e., $\triangle=\{(x, x) \mid x \in X\} \subset E(G)$. We say that a mapping $f: X \rightarrow X$ is a G-contraction if $f$ preserves edges of $G$, i.e., for every $x, y \in X$,

$$
(x, y) \in E(G) \quad \Rightarrow \quad(f(x), f(y)) \in E(G)
$$

and there exists $\alpha \in(0,1)$ such that $x, y \in X$,

$$
(x, y) \in E(G) \quad \Rightarrow \quad d(f(x), f(y)) \leq \alpha d(x, y) .
$$

The mapping $f: X \rightarrow X$ satisfying condition (1) is also called a graph-preserving mapping. Jachymski showed in [17] that under some properties on $X$, a $G$-contraction $f: X \rightarrow$ $X$ has a fixed point if and only if there exists $x \in X$ such that $(x, f(x)) \in E(G)$.

Recently, Beg and Butt [18] introduced the concept of ' $G$-contraction' for a multi-valued mapping $T: X \rightarrow C B(X)$ defined as follows.

Definition 1.7 Let $T: X \rightarrow C B(X)$ be a multi-valued mapping. The mapping $T$ is said to be a $G$-contraction if there exists $k \in(0,1)$ such that

$$
H(T x, T y) \leq k d(x, y)
$$

for all $(x, y) \in E(G)$ and if $u \in T x$ and $v \in T y$ are such that

$$
d(u, v) \leq k d(x, y)+\alpha
$$

for each $\alpha>0$, then $(u, v) \in E(G)$. 
They showed that if $(X, d)$ is a complete metric space and $(X, d)$ has Property A [18], then G-contraction mapping $T: X \rightarrow C B(X)$ has a fixed point if and only if there exist $x \in X$ and $y \in T x$ such that $(x, y) \in E(G)$.

In 2011, Nicolae, O'Regan, and Petrusel [19] extended the notion of multi-valued contraction on a metric space with a graph in considering the fixed point shown below.

Theorem 1.8 Let $F: X \rightarrow X$ be a multi-valued map with nonempty closed values. Assume that

(1) there exists $\lambda \in(0,1)$ such that $D(F(x), F(y)) \leq \lambda d(x, y)$ for all $(x, y) \in E(G)$;

(2) for each $(x, y) \in E(G)$, each $u \in F(x)$ and $v \in F(y)$ satisfying $d(u, v) \leq a d(x, y)$ for some $a \in(0,1),(u, v) \in E(G)$ holds;

(3) $X$ has Property A.

If there exist $x_{0}, x_{1} \in X$ such that $x_{1} \in\left[x_{0}\right]_{G}^{1} \cap F\left(x_{0}\right)$, then $F$ has a fixed point.

In 2013, Dinevari and Frigon [20] introduced a concept of 'G-contraction' which is weaker than that of Beg and Butt [18] and weaker than that of Nicolae, O'Regan, and Petrusel [19].

Definition 1.9 Let $T: X \rightarrow 2^{X}$ be a map with nonempty values. We say that $T$ is a $G$ contraction (in the sense of Dinevari and Frigon) if there exists $\alpha \in(0,1)$ such that for all $(x, y) \in E(G)$ and all $u \in T x$, there exists $v \in T y$ such that $(u, v) \in E(G)$ and $d(u, v) \leq \alpha d(x, y)$.

They showed that under some properties, weaker than Property A, on a metric space a multi-valued G-contraction with the closed value has a fixed point.

Most recently, Tiammee and Suantai [21] introduced the concept of 'graph preserving' for multi-valued mappings and proved their fixed point theorem in a complete metric space endowed with a graph.

Definition 1.10 [21] Let $X$ be a nonempty set, $G=(V(G), E(G))$ a directed graph such that $V(G)=X$, and $T: X \rightarrow C B(X) . T$ is said to be graph preserving if

$$
(x, y) \in E(G) \quad \Rightarrow \quad(u, v) \in E(G)
$$

for all $u \in T x$ and $v \in T y$.

Definition 1.11 [21] Let $X$ be a nonempty set, $G=(V(G), E(G))$ a directed graph such that $V(G)=X, g: X \rightarrow X$, and $T: X \rightarrow C B(X)$. $T$ is said to be $g$-graph preserving if for any $x, y \in X$, such that

$$
(g(x), g(y)) \in E(G) \quad \Rightarrow \quad(u, v) \in E(G)
$$

for all $u \in T x$ and $v \in T y$.

Definition 1.12 Let $(X, d)$ be a metric space, $G=(V(G), E(G))$ a directed graph such that $V(G)=X, g: X \rightarrow X$, and $T: X \rightarrow C B(X) . T$ is said to be a multi-valued weak $G$-contraction with respect to $g$ or a $(g, \alpha, L)$-G-contraction if there exists a function 
$\alpha:[0, \infty) \rightarrow[0,1)$ satisfying

$$
\limsup _{r \rightarrow t^{+}} \alpha(r)<1
$$

for every $t \in[0, \infty)$ and a nonnegative number $L$ with

$$
H(T x, T y) \leq \alpha(d(g(x), g(y))) d(g(x), g(y))+L D(g(y), T x)
$$

for all $x, y \in X$ such that $(g(x), g(y)) \in E(G)$.

Theorem 1.13 [21] Let $(X, d)$ be a complete metric space, $G=(V(G), E(G))$ a directed graph such that $V(G)=X$, and $g: X \rightarrow X$ a surjective mapping. If $T: X \rightarrow C B(X)$ is a multi-valued mapping satisfying the following properties:

(1) $T$ is a g-graph preserving mapping;

(2) there exists $x_{0} \in X$ such that $\left(g\left(x_{0}\right), y\right) \in E(G)$ for some $y \in T x_{0}$;

(3) $X$ has Property A;

(4) $T$ is a $(g, \alpha, L)-G$-contraction,

then there exists $u \in X$ such that $g(u) \in T u$.

The condition of $T$ in Definition 1.11 to be $g$-graph preserving requires all pairs $(u, v)$ where $u \in T x$ and $v \in T y$ have connecting edges whenever $(g(x), g(y)) \in E(G)$. With some modification, we are interested in proposing the new concept of ' $g$-graph preserving' for multi-valued mappings in a complete metric space endowed with a graph and the fixed point theorem is also determined.

\section{Preliminaries}

Let $(X, d)$ be a metric space. For $x \in X$ and $A, B \in \operatorname{Comp}(X)$, define

$$
\begin{aligned}
& d(x, A)=\inf \{d(x, y) \mid y \in A\}, \\
& D(A, B)=\inf \{d(x, B) \mid x \in A\} .
\end{aligned}
$$

For each $a \in A$, define

$$
P_{B}(a)=\{b \in B \mid d(a, b)=d(a, B)\} .
$$

Each element in $P_{B}(a)$ is called a projection point of $a$ into $B$. Note that if $B$ is compact, then $P_{B}(a)$ is always a nonempty set. Also, define

$$
H(A, B)=\max \left\{\sup _{x \in B} d(x, A), \sup _{x \in A} d(x, B)\right\} .
$$

The mapping $H$ is said to be a Hausdorff metric induced by $d$. The next two lemmas will play central roles in our main results.

Lemma 2.1 [12] Let $(X, d)$ be a metric space. If $A, B \in \operatorname{Comp}(X)(\operatorname{or} C B(X))$ and $x \in A$, then for each $\epsilon>0$, there is $b \in B$ such that

$$
d(a, b) \leq H(A, B)+\epsilon .
$$


Lemma 2.2 [15] Let $(X, d)$ be a metric space, $\left\{A_{k}\right\} \subset \operatorname{Comp}(X)(\operatorname{or} C B(X)),\left\{x_{k}\right\}$ a sequence in $X$ such that $x_{k} \in A_{k-1}$, and $\alpha:[0, \infty) \rightarrow[0,1)$ a function satisfying $\lim \sup _{r \rightarrow t^{+}} \alpha(r)<1$ for every $t \in[0, \infty)$. Suppose that $d\left(x_{k-1}, x_{k}\right)$ is a non-increasing sequence such that

$$
\begin{aligned}
& H\left(A_{k-1}, A_{k}\right) \leq \alpha\left(d\left(x_{k-1}, x_{k}\right)\right) d\left(x_{k-1}, x_{k}\right), \\
& d\left(x_{k}, x_{k+1}\right) \leq H\left(A_{k-1}, A_{k}\right)+\left(\alpha\left(d\left(x_{k-1}, x_{k}\right)\right)\right)^{n_{k}},
\end{aligned}
$$

where $\left\{n_{k}\right\}$ is an increasing sequence and $k, n_{k} \in \mathbb{N}$. Then $\left\{x_{k}\right\}$ is a Cauchy sequence in $X$.

Next we will give notions and examples of new types of multi-valued mapping with compact value which are weaker than that of Tiammee and Suantai [21].

Definition 2.3 Let $X$ be a nonempty set and $G=(V(G), E(G))$ be a directed graph such that $V(G)=X$, and $T: X \rightarrow \operatorname{Comp}(X) . T$ is said to be weak graph preserving if it satisfies the following:

for each $x, y \in X$, if $(x, y) \in E(G)$, then for each $u \in T x$ there is $v \in P_{T y}(u)$ such that $(u, v) \in E(G)$.

In Example 2.4, we illustrate a mapping $T$ which is weak graph preserving but not graph preserving.

Example 2.4 Let $\mathbb{N}$ be a metric space with the usual metric and $G=(\mathbb{N}, E(G))$ where

$$
E(G)=\{(2 n-1,2 n): n \in \mathbb{N}\} \cup\{(2 n, 1): n \in \mathbb{N}\} .
$$

Define $T: \mathbb{N} \rightarrow \operatorname{Comp}(X)$ by

$$
T x= \begin{cases}\{2 k, 2 k+2\} & \text { if } x=2 k-1, k \in \mathbb{N} \\ \{1\} & \text { if } x=2 k, k \in \mathbb{N} .\end{cases}
$$

We will show that $T$ is weak graph preserving. If $(x, y)=(2 k-1,2 k)$ where $k \in \mathbb{N}$, then $T x=\{2 k, 2 k+2\}$ and $T y=\{1\}$. We can see that $P_{T y}(2 k)=\{1\}=P_{T y}(2 k+2)$ and $(2 k, 1),(2 k+$ $2,1) \in E(G)$.

If $(x, y)=(2 k, 1)$ where $k \in \mathbb{N}$, then $T x=\{1\}$ and $T y=\{2,4\}$. We can see that $P_{T y}(1)=\{2\}$ and $(1,2) \in E(G)$. It is easy to see that $(1,4) \notin E(G)$ and so $T$ is not graph preserving.

Next we will give an another example of a weak graph-preserving mapping that is not a $G$-contraction in the sense of Dinevari and Frigon [20].

Example 2.5 Let $X=\{1,2,3,4,6,8\}$ be a metric space with the usual metric and $G=$ $(X, E(G))$ where

$$
E(G)=\{(1,1),(1,3),(2,2),(2,6),(4,2),(4,4),(4,6)\}
$$


Define $T: X \rightarrow \operatorname{Comp}(X)$ by

$$
T x= \begin{cases}\{2,4\} & \text { if } x=1,4 \\ \{6,8\} & \text { if } x=3 \\ \{2\} & \text { if } x=2,6,8\end{cases}
$$

We will show that $T$ is weak graph preserving. If $(x, y)=(1,1)$ then $T x=\{2,4\}$ and $T y=$ $\{2,4\}$. We can see that $P_{T y}(2)=\{2\}, P_{T y}(4)=\{4\}$ and $(2,2),(4,4) \in E(G)$.

If $(x, y)=(1,3)$ then $T x=\{2,4\}$ and $T y=\{6,8\}$. We can see that $P_{T y}(2)=\{6\}, P_{T y}(4)=\{6\}$, and $(2,6),(4,6) \in E(G)$.

If $(x, y)=(2,2)$ or $(x, y)=(2,6)$ then $T x=\{2\}$ and $T y=\{2\}$. We can see that $P_{T y}(2)=\{2\}$ and $(2,2) \in E(G)$.

If $(x, y)=(4,2)$ or $(x, y)=(4,6)$ then $T x=\{2,4\}$ and $T y=\{2\}$. We can see that $P_{T y}(2)=$ $\{2\}=P_{T y}(4)$ and $(2,2),(4,2) \in E(G)$.

So, $T$ is weak graph preserving but it is not a $G$-contraction in the sense of Dinevari and Frigon since for each $u \in T x$ and $v \in T y$ with $(x, y)=(1,3), d(u, v) \geq 2>\alpha d(1,3)$ for all $\alpha \in(0,1)$.

Definition 2.6 Let $X$ be a nonempty set, $G=(V(G), E(G))$ a directed graph such that $V(G)=X, T: X \rightarrow \operatorname{Comp}(X)$, and $g: X \rightarrow X . T$ is said to be weak g-graph preserving if it satisfies the following:

for each $x, y \in X$, if $(g(x), g(y)) \in E(G)$, then for each $u \in T x$ there is $v \in P_{T y}(u)$ such that $(u, v) \in E(G)$.

Example 2.7 Let $\mathbb{N}$ be a metric space with the usual metric, $G=(\mathbb{N}, E(G))$ where

$$
E(G)=\{(2 n-1,2 n): n \in \mathbb{N}\} \cup\{(2 n, 1): n \in \mathbb{N}\} .
$$

Define $T: \mathbb{N} \rightarrow \operatorname{Comp}(X)$ by

$$
T x= \begin{cases}\{2 k, 2 k+2\} & \text { if } x=2 k-1, k \in \mathbb{N} \\ \{1\} & \text { if } x=2 k, k \in \mathbb{N}\end{cases}
$$

and $g: X \rightarrow X$ by

$$
g(x)= \begin{cases}2 k & \text { if } x=2 k+2, k \in \mathbb{N} \\ 2 k-1 & \text { if } x=2 k+1, k \in \mathbb{N} \\ 1 & \text { if } x=1 \\ 2 & \text { if } x=2\end{cases}
$$

We will show that $T$ is weak $g$-graph preserving.

If $(g(x), g(y))=(1,2)$, then $(x, y)=(1,2)$ and $T x=\{2,4\}$ and $T y=\{1\}$. We can see that $P_{T y}(2)=\{1\}=P_{T y}(4)$ and $(2,1),(4,1) \in E(G)$.

If $(g(x), g(y))=(2,1)$, then $(x, y)=(2,1)$ and $T x=\{1\}$ and $T y=\{2,4\}$. We can see that $P_{T y}(1)=\{2\}$ and $(1,2) \in E(G)$. 
If $(g(x), g(y))=(2 k-1,2 k)$ where $k \in \mathbb{N} \backslash\{1\}$, then $(x, y)=(2 k+1,2 k+2)$ and $T x=\{2 k+$ $2,2 k+4\}$ and $T y=\{1\}$. We can see that $P_{T y}(2 k+2)=\{1\}=P_{T y}(2 k+4)$ and $(2 k+2,1),(2 k+$ $4,1) \in E(G)$.

If $(g(x), g(y))=(2 k, 1)$ where $k \in \mathbb{N} \backslash\{1\}$, then $(x, y)=(2 k+2,1)$ and $T x=\{1\}$ and $T y=$ $\{2,4\}$. We can see that $P_{T y}(1)=\{2\}$ and $(1,2) \in E(G)$. Hence $T$ is weak $g$-graph preserving.

\section{Main results}

We first recall Property A before the main theorem is proved.

Property A For any sequence $\left\{x_{n}\right\}$ in $X$, if $x_{n} \rightarrow x$ and $\left(x_{n}, x_{n+1}\right) \in E(G)$ for $n \in \mathbb{N}$, then there is a subsequence $\left\{x_{n_{k}}\right\}$ such that $\left(x_{n_{k}}, x\right) \in E(G)$ for $k \in \mathbb{N}$.

Theorem 3.1 Let $(X, d)$ be a complete metric space, $G=(V(G), E(G))$ a directed graph such that $V(G)=X$, and $g: X \rightarrow X$ a surjective map. If $T: X \rightarrow \operatorname{Comp}(X)$ is a multi-valued mapping satisfying the following properties:

(1) $T$ is weak g-graph preserving;

(2) $X_{T}=\{x \in X \mid(g(x), y) \in E(G)$ for some $y \in T x\} \neq \emptyset$;

(3) $X$ has Property A;

(4) $T$ is a $(g, \alpha, L)$-G-contraction,

then there exists $u \in X$ such that $g(u) \in T u$.

Proof By (2), let $x_{0} \in X_{T}$. Then there exists $y_{1} \in T x_{0}$ such that $\left(g\left(x_{0}\right), y_{1}\right) \in E(G)$. Since $g$ is surjective, there exists $x_{1} \in X$ such that $y_{1}=g\left(x_{1}\right)$. Thus we have $\left(g\left(x_{0}\right), g\left(x_{1}\right)\right) \in E(G)$. Since $\left[\alpha\left(d\left(g\left(x_{0}\right), g\left(x_{1}\right)\right)\right)\right]^{n} \rightarrow 0$, there exists $n_{1} \in \mathbb{N}$ such that

$$
\left[\alpha\left(d\left(g\left(x_{0}\right), g\left(x_{1}\right)\right)\right)\right]^{n_{1}} \leq\left[1-\alpha\left(d\left(g\left(x_{0}\right), g\left(x_{1}\right)\right)\right)\right] d\left(g\left(x_{0}\right), g\left(x_{1}\right)\right) .
$$

Since $T x_{1}$ is compact, it follows that $P_{T x_{1}}\left(g\left(x_{1}\right)\right) \neq \emptyset$. Since $T$ is weak $g$-graph preserving, there exists $y_{2} \in P_{T x_{1}}\left(g\left(x_{1}\right)\right)$ such that $\left(g\left(x_{1}\right), y_{2}\right) \in E(G)$ and $d\left(g\left(x_{1}\right), y_{2}\right)=d\left(g\left(x_{1}\right), T x_{1}\right)$. Again since $g$ is surjective, there is $x_{2} \in X$ such that $g\left(x_{2}\right)=y_{2}$. By Lemma 2.1, there is $y_{1}^{\prime} \in T x_{1}$ such that

$$
d\left(g\left(x_{1}\right), y_{1}^{\prime}\right) \leq H\left(T x_{0}, T x_{1}\right)+\left[\alpha\left(d\left(g\left(x_{0}\right), g\left(x_{1}\right)\right)\right)\right]^{n_{1}} .
$$

It follows that

$$
\begin{aligned}
d\left(g\left(x_{1}\right), g\left(x_{2}\right)\right) & =d\left(g\left(x_{1}\right), T x_{1}\right) \\
& \leq d\left(g\left(x_{1}\right), y_{1}^{\prime}\right) \\
& \leq H\left(T x_{0}, T x_{1}\right)+\left[\alpha\left(d\left(g\left(x_{0}\right), g\left(x_{1}\right)\right)\right)\right]^{n_{1}} .
\end{aligned}
$$

Since $T$ is a $(g, \alpha, L)$-G-contraction and $\left(g\left(x_{1}\right), g\left(x_{2}\right)\right) \in E(G)$, we have

$$
\begin{aligned}
d\left(g\left(x_{1}\right), g\left(x_{2}\right)\right) \leq & H\left(T x_{0}, T x_{1}\right)+\left[\alpha\left(d\left(g\left(x_{0}\right), g\left(x_{1}\right)\right)\right)\right]^{n_{1}} \\
\leq & \alpha\left(d\left(g\left(x_{0}\right), g\left(x_{1}\right)\right)\right) d\left(g\left(x_{0}\right), g\left(x_{1}\right)\right)+L D\left(g\left(x_{1}\right), T x_{0}\right) \\
& +\left[\alpha\left(d\left(g\left(x_{0}\right), g\left(x_{1}\right)\right)\right)\right]^{n_{1}}
\end{aligned}
$$




$$
\begin{aligned}
& \leq \alpha\left(d\left(g\left(x_{0}\right), g\left(x_{1}\right)\right)\right) d\left(g\left(x_{0}\right), g\left(x_{1}\right)\right)+\left[\alpha\left(d\left(g\left(x_{0}\right), g\left(x_{1}\right)\right)\right)\right]^{n_{1}} \\
& \leq d\left(g\left(x_{0}\right), g\left(x_{1}\right)\right) .
\end{aligned}
$$

Next, since $\left[\alpha\left(d\left(g\left(x_{1}\right), g\left(x_{2}\right)\right)\right)\right]^{n} \rightarrow 0$, there exists $n_{2} \in \mathbb{N}$ such that $n_{2}>n_{1}$ and

$$
\left[\alpha\left(d\left(g\left(x_{1}\right), g\left(x_{2}\right)\right)\right)\right]^{n_{2}} \leq\left[1-\alpha\left(d\left(g\left(x_{1}\right), g\left(x_{2}\right)\right)\right)\right] d\left(g\left(x_{1}\right), g\left(x_{2}\right)\right) .
$$

Since $T x_{2}$ is compact, it follows that $P_{T x_{2}}\left(g\left(x_{2}\right)\right) \neq \emptyset$. Since $T$ is weak $g$-graph preserving, there exists $y_{3} \in P_{T x_{2}}\left(g\left(x_{2}\right)\right)$ such that $\left(g\left(x_{2}\right), y_{3}\right) \in E(G)$ and $d\left(g\left(x_{2}\right), y_{3}\right)=d\left(g\left(x_{2}\right), T x_{2}\right)$. Again since $g$ is surjective, there is $x_{3} \in X$ such that $g\left(x_{3}\right)=y_{3}$. By Lemma 2.1, there is $y_{2}^{\prime} \in T x_{2}$ such that

$$
d\left(g\left(x_{1}\right), y_{2}^{\prime}\right) \leq H\left(T x_{1}, T x_{2}\right)+\left[\alpha\left(d\left(g\left(x_{1}\right), g\left(x_{2}\right)\right)\right)\right]^{n_{2}} .
$$

It follows that

$$
\begin{aligned}
d\left(g\left(x_{2}\right), g\left(x_{3}\right)\right) & =d\left(g\left(x_{2}\right), T x_{2}\right) \\
& \leq d\left(g\left(x_{2}\right), y_{2}^{\prime}\right) \\
& \leq H\left(T x_{1}, T x_{2}\right)+\left[\alpha\left(d\left(g\left(x_{1}\right), g\left(x_{2}\right)\right)\right)\right]^{n_{2}} .
\end{aligned}
$$

Since $T$ is a $(g, \alpha, L)$-G-contraction and $\left(g\left(x_{2}\right), g\left(x_{3}\right)\right) \in E(G)$, we have

$$
\begin{aligned}
d\left(g\left(x_{2}\right), g\left(x_{3}\right)\right) \leq & H\left(T x_{1}, T x_{2}\right)+\left[\alpha\left(d\left(g\left(x_{1}\right), g\left(x_{2}\right)\right)\right)\right]^{n_{2}} \\
\leq & \alpha\left(d\left(g\left(x_{1}\right), g\left(x_{2}\right)\right)\right) d\left(g\left(x_{1}\right), g\left(x_{2}\right)\right)+L D\left(g\left(x_{2}\right), T x_{1}\right) \\
& +\left[\alpha\left(d\left(g\left(x_{1}\right), g\left(x_{2}\right)\right)\right)\right]^{n_{2}} \\
\leq & \alpha\left(d\left(g\left(x_{1}\right), g\left(x_{2}\right)\right)\right) d\left(g\left(x_{1}\right), g\left(x_{2}\right)\right)+\left[\alpha\left(d\left(g\left(x_{1}\right), g\left(x_{2}\right)\right)\right)\right]^{n_{2}} \\
\leq & d\left(g\left(x_{1}\right), g\left(x_{2}\right)\right) .
\end{aligned}
$$

Continuing in this process, we produce a sequence $\left\{g\left(x_{k}\right)\right\}$ in $X$ and an increasing sequence $\left\{n_{k}\right\}$ in $\mathbb{N}$ such that for each $k \in \mathbb{N}, g\left(x_{k+1}\right) \in T x_{k},\left(g\left(x_{k}\right), g\left(x_{k+1}\right)\right) \in E(G)$,

$$
\begin{aligned}
& {\left[\alpha\left(d\left(g\left(x_{k-1}\right), g\left(x_{k}\right)\right)\right)\right]^{n_{k}} \leq\left[1-\alpha\left(d\left(g\left(x_{k-1}\right), g\left(x_{k}\right)\right)\right)\right] d\left(g\left(x_{k-1}\right), g\left(x_{k}\right)\right),} \\
& H\left(T x_{k-1}, T x_{k}\right) \leq \alpha\left(d\left(g\left(x_{k-1}\right), g\left(x_{k}\right)\right)\right) d\left(g\left(x_{k-1}\right), g\left(x_{k}\right)\right),
\end{aligned}
$$

and

$$
d\left(g\left(x_{k}\right), g\left(x_{k+1}\right)\right) \leq H\left(T x_{k-1}, T x_{k}\right)+\left[\alpha\left(d\left(g\left(x_{k-1}\right), g\left(x_{k}\right)\right)\right)\right]^{n_{k}} .
$$

We note that

$$
\begin{aligned}
d\left(g\left(x_{k}\right), g\left(x_{k+1}\right)\right) & \leq H\left(T x_{k-1}, T x_{k}\right)+\left[\alpha\left(d\left(g\left(x_{k-1}\right), g\left(x_{k}\right)\right)\right)\right]^{n_{k}} \\
& \leq \alpha\left(d\left(g\left(x_{k-1}\right), g\left(x_{k}\right)\right)\right) d\left(g\left(x_{k-1}\right), g\left(x_{k}\right)\right)
\end{aligned}
$$




$$
\begin{aligned}
& +\left[\alpha\left(d\left(g\left(x_{k-1}\right), g\left(x_{k}\right)\right)\right)\right]^{n_{k}} \\
\leq & d\left(g\left(x_{k-1}\right), g\left(x_{k}\right)\right) .
\end{aligned}
$$

Hence, $d\left(g\left(x_{k}\right), g\left(x_{k+1}\right)\right) \leq d\left(g\left(x_{k-1}\right), g\left(x_{k}\right)\right)$ and so $\left\{d\left(g\left(x_{k}\right), g\left(x_{k+1}\right)\right)\right\}$ is a non-increasing sequence. By Lemma 2.2, $\left\{g\left(x_{k}\right)\right\}$ is a Cauchy sequence in $X$. Since $X$ is complete, the sequence $\left\{g\left(x_{k}\right)\right\}$ converges to a point $g(u)$ for some $u \in X$. By Property A in (3), there is a subsequence $\left\{g\left(x_{k_{n}}\right)\right\}$ such that $\left(g\left(x_{k_{n}}\right), g(u)\right) \in E(G)$ for any $n \in \mathbb{N}$. Claim that $g(u) \in T u$. Note that for each $g\left(x_{k_{n}+1}\right)$,

$$
\begin{aligned}
D(g(u), T u) \leq & d\left(g(u), g\left(x_{k_{n}+1}\right)\right)+D\left(g\left(x_{k_{n}+1}\right), T u\right) \\
\leq & d\left(g(u), g\left(x_{k_{n}+1}\right)\right)+H\left(T x_{k_{n}}, T u\right) \\
\leq & d\left(g(u), g\left(x_{k_{n}+1}\right)\right)+\alpha\left(d\left(g\left(x_{k_{n}}\right), g(u)\right)\right) d\left(g\left(x_{k_{n}}\right), g(u)\right) \\
& +L D\left(g(u), T x_{k_{n}}\right) \\
\leq & d\left(g(u), g\left(x_{k_{n}+1}\right)\right)+\alpha\left(d\left(g\left(x_{k_{n}}\right), g(u)\right)\right) d\left(g\left(x_{k_{n}}\right), g(u)\right) \\
& +L d\left(g(u), g\left(x_{k_{n}+1}\right)\right) .
\end{aligned}
$$

Since $g\left(x_{k_{n}}\right)$ converges to $g(u)$ as $n \rightarrow \infty$, it follows that $D(g(u), T u)=0$. Since $T u$ is compact, we conclude that $g(u) \in T u$, completing the proof.

Remark 3.2 Theorem 3.1 is an extension of Theorem 1.13 in the case of a mapping $T$ : $X \rightarrow C B(X)$ having compact values.

A partial order is a binary relation $\leq$ over the set $X$ which satisfies the following conditions:

(1) $x \leq x$ (reflexivity);

(2) if $x \leq y$ and $y \leq x$, then $x=y$ (antisymmetry);

(3) if $x \leq y$ and $y \leq z$, then $x \leq z$ (transitivity),

for all $x, y \in X$. A set with a partial order $\leq$ is called a partially ordered set. We write $x<y$ if $x \leq y$ and $x \neq y$.

Definition 3.3 Let $(X, \leq)$ be a partially ordered set. For each $A, B \subset X, A \prec B$ if $a \leq b$ for any $a \in A, b \in B$.

Definition 3.4 [21] Let $(X, d)$ be a metric space endowed with a partial order $\leq, g: X \rightarrow X$ a surjective map, and $T: X \rightarrow \operatorname{Comp}(X) . T$ is said to be $g$-increasing if for any $x, y \in X$,

$$
g(x)<g(y) \quad \Rightarrow \quad T x \prec T y .
$$

In the case $g$ is the identity map, the mapping $T$ is called an increasing mapping.

Corollary 3.5 Let $(X, d)$ be a metric space endowed with a partial order $\leq, g: X \rightarrow X a$ surjective map and $T: X \rightarrow \operatorname{Comp}(X)$ a multi-valued mapping. Suppose that

(1) $T$ is $g$-increasing;

(2) there exist $x_{0} \in X$ and $u \in T x_{0}$ such that $g\left(x_{0}\right)<u$; 
(3) for each sequence $\left\{x_{k}\right\}$ such that $g\left(x_{k}\right)<g\left(x_{k+1}\right)$ for all $k \in \mathbb{N}$ and $g\left(x_{k}\right)$ converges to $g(x)$ for some $x \in X$, then $g\left(x_{k}\right)<g(x)$ for all $k \in \mathbb{N}$;

(4) there exist a function $\alpha:[0, \infty) \rightarrow[0,1)$ satisfying $\lim \sup _{r \rightarrow t^{+}} \alpha(r)<1$ for every $t \in[0, \infty)$ and a nonnegative number $L$ with

$$
H(T x, T y) \leq \alpha(d(g(x), g(y))) d(g(x), g(y))+L D(g(y), T x)
$$

for all $x, y \in X$ such that $g(x)<g(y)$;

(5) the metric $d$ is complete.

Then there exists $u \in X$ such that $g(u) \in T u$.

Proof Define $G=(V(G), E(G))$ where $V(G)=X$ and $E(G)=\{(x, y) \mid x<y\}$. Let $x, y \in X$ be such that $(g(x), g(y)) \in E(G)$. Then $g(x)<g(y)$ so by (1) it implies that $T x \prec T y$. For each $u \in$ $T x, v \in T y, u<v$. Since $T y$ is compact, it follows that $P_{T y}(x) \neq \emptyset$ and $P_{T y}(x) \subset T y$ for all $x \in$ $T x$. Thus $(x, v) \in E(G)$ for all $v \in P_{T y}(x)$ and all $x \in T x$. That is, $T$ is weak $g$-graph preserving. By assumption (2), there exist $x_{0} \in X$ and $u \in T x_{0}$ such that $g\left(x_{0}\right)<u$. So $\left(g\left(x_{0}\right), u\right) \in E(G)$ and hence the condition (2) in Theorem 3.1 is satisfied. Moreover, the conditions (3) and (4) in Theorem 3.1 are also satisfied. Therefore the result of this corollary is followed by Theorem 3.1.

Theorem 3.6 Let $(X, d)$ be a metric space endowed with a partial order $\leq$ and $T: X \rightarrow$ $\operatorname{Comp}(X)$ a multi-valued mapping. Suppose that

(1) $T$ is increasing;

(2) there exist $x_{0} \in X$ and $u \in T x_{0}$ such that $x_{0}<u$;

(3) for each sequence $\left\{x_{k}\right\}$ such that $x_{k}<x_{k+1}$ for all $k \in \mathbb{N}$ and $x_{k}$ converges to $x$ for some $x \in X$, then $x_{k}<x$ for all $k \in \mathbb{N}$;

(4) there exist a function $\alpha:[0, \infty) \rightarrow[0,1)$ satisfying $\lim \sup _{r \rightarrow t^{+}} \alpha(r)<1$ for every $t \in[0, \infty)$ and a nonnegative number $L$ with

$$
H(T x, T y) \leq \alpha(d(x, y)) d(x, y)+L D(y, T x)
$$

for all $x, y \in X$ with $x<y$;

(5) the metric $d$ is complete.

Then there exists $u \in X$ such that $u \in$ Tu. Furthermore, if $\sup \{\alpha(r): r \in[0, \infty)\}<1$ and $L<1-\sup \{\alpha(r): r \in[0, \infty)\}$, then $T$ has a unique fixed point.

Proof Setting $g(x)=x$, by Corollary 3.5 we have $\operatorname{Fix}(T) \neq \emptyset$. With conditions $\sup \{\alpha(r): r \in$ $[0, \infty)\}<1$ and $L<1-\sup \{\alpha(r): r \in[0, \infty)\}$, we will show that $T$ has a unique fixed point. Let $u, v \in \operatorname{Fix}(T)$. Suppose to a contrary that $u \neq v$. Without loss of generality, assume that $u<v$. By the condition (4), we have

$$
\begin{aligned}
d(u, v) & \leq H(T u, T v) \\
& \leq \alpha(d(u, v)) d(u, v)+L D(v, T u) \\
& \leq \sup \{\alpha(r): r \in[0, \infty)\} d(u, v)+L d(u, v) \\
& =(\sup \{\alpha(r): r \in[0, \infty)\}+L) d(u, v) .
\end{aligned}
$$


Since $\sup \{\alpha(r): r \in[0, \infty)\}+L<1$, this yields $d(u, v)<d(u, v)$, a contradiction. Therefore $u=v$, which implies that $T$ has a unique fixed point.

Next we give an example such that $T$ has a unique fixed point but $T$ is neither a graph-preserving nor a multi-valued G-contraction in the sense of Nicolae, O'Regan, and Petrusel [19].

Example 3.7 Let $X=\{0\} \cup\left\{\frac{1}{2^{n}}: n \in \mathbb{N} \cup\{0\}\right\}$ be a metric space with the usual metric $d$. Consider the directed graph defined by $V(G)=X$ and

$$
E(G)=\left\{\left(\frac{1}{2^{n}}, \frac{1}{2^{n+1}}\right),\left(\frac{1}{2^{n}}, 0\right): n \in \mathbb{N} \cup\{0\}\right\} \cup \Delta,
$$

where $\Delta$ is the diagonal in $X \times X$. Let $T: X \rightarrow \operatorname{Comp}(X)$ be defined by

$$
T x= \begin{cases}\{0,1\} & \text { if } x=0 \\ \left\{\frac{1}{2^{n+1}}, 1\right\} & \text { if } x=\frac{1}{2^{n}}, n \in \mathbb{N} \\ \left\{\frac{1}{2}\right\} & \text { if } x=1\end{cases}
$$

Then:

(1) $T$ has a fixed point;

(2) $T$ is not graph preserving as defined by Tiammee and Suantai [21];

(3) $T$ is not a G-contraction in the sense of Nicolae, O'Regan, and Petrusel [19].

Proof (1) We will show that $T$ is a $(\alpha, g, L)$-G-contraction with $\alpha \geq \frac{1}{2}, L \geq 2$, and $g=i_{X}$. Let $(x, y) \in E(G)$.

If $(x, y)=\left(1, \frac{1}{2}\right)$, then $T x=\left\{\frac{1}{2}\right\}, T y=\left\{\frac{1}{4}, 1\right\}$, and

$$
H(T x, T y)=\frac{1}{4}=\frac{1}{2} d(x, y) \leq \alpha(d(x, y)) d(x, y)+L D(y, T x) .
$$

If $(x, y)=(1,0)$, then $T x=\left\{\frac{1}{2}\right\}, T y=\{0,1\}$, and

$$
H(T x, T y)=\frac{1}{2}=\frac{1}{2} d(x, y) \leq \alpha(d(x, y)) d(x, y)+L D(y, T x) .
$$

If $(x, y)=\left(\frac{1}{2^{n}}, \frac{1}{2^{n+1}}\right)$ for all $n \in \mathbb{N}$, then $T x=\left\{\frac{1}{2^{n+1}}, 1\right\}$ and $T y=\left\{\frac{1}{2^{n+2}}, 1\right\}$. It is easy to check that

$$
\begin{aligned}
H(T x, T y) & =\max \left\{\sup _{a \in T y} d(a, T x), \sup _{b \in T x} d(b, T y)\right\} \\
& =d\left(\frac{1}{2^{n+1}}, \frac{1}{2^{n+2}}\right) \\
& =\frac{1}{2} d\left(\frac{1}{2^{n}}, \frac{1}{2^{n+1}}\right) \\
& =\frac{1}{2} d(x, y) \\
& \leq \alpha(d(x, y)) d(x, y)+L D(y, T x) .
\end{aligned}
$$


If $(x, y)=\left(\frac{1}{2^{n}}, 0\right)$ for all $n \in \mathbb{N}$, then $T x=\left\{\frac{1}{2^{n+1}}, 1\right\}$ and $T y=\{0,1\}$. We have

$$
\begin{aligned}
H(T x, T y) & =\max \left\{\sup _{a \in T y} d(a, T x), \sup _{b \in T x} d(b, T y)\right\} \\
& =\frac{1}{2^{n+1}} \\
& =\frac{1}{2} d\left(\frac{1}{2^{n}}, 0\right) \\
& =\frac{1}{2} d(x, y) \\
& \leq \alpha(d(x, y)) d(x, y)+L D(y, T x) .
\end{aligned}
$$

If $(x, y)=(0,0)$ and $(x, y)=\left(\frac{1}{2^{n}}, \frac{1}{2^{n}}\right)$, then $T x=T y$ which obviously implies that $H(T x, T y)=$ 0 . So,

$$
H(T x, T y) \leq \alpha(d(x, y)) d(x, y)+L D(y, T x)
$$

Hence $T$ is a $(\alpha, g, L)$-G-contraction. Next, we will show that $T$ is weak graph preserving. Let $(x, y) \in E(G)$.

If $(x, y)=\left(1, \frac{1}{2}\right)$, then $T x=\left\{\frac{1}{2}\right\}, T y=\left\{\frac{1}{4}, 1\right\}$, and $P_{T y}\left(\frac{1}{2}\right)=\left\{\frac{1}{4}\right\}$ and $\left(\frac{1}{2}, \frac{1}{4}\right) \in E(G)$.

If $(x, y)=(1,0)$, then $T x=\left\{\frac{1}{2}\right\}, T y=\{0,1\}$, and $P_{T y}\left(\frac{1}{2}\right)=\{0,1\}$ and $\left(\frac{1}{2}, 0\right) \in E(G)$.

If $(x, y)=\left(\frac{1}{2^{n}}, \frac{1}{2^{n+1}}\right)$ for all $n \in \mathbb{N}$, then $T x=\left\{\frac{1}{2^{n+1}}, 1\right\}$ and $T y=\left\{\frac{1}{2^{n+2}}, 1\right\}$. It is easy to see that $P_{T y}\left(\frac{1}{2^{n+1}}\right)=\left\{\frac{1}{2^{n+2}}\right\}, P_{T y}(1)=\{1\}$, and $\left(\frac{1}{2^{n+1}}, \frac{1}{2^{n+2}}\right),(1,1) \in E(G)$.

If $(x, y)=\left(\frac{1}{2^{n}}, 0\right)$ for all $n \in \mathbb{N}$, then $T x=\left\{\frac{1}{2^{n+1}}, 1\right\}$ and $T y=\{0,1\}$. We have $P_{T y}\left(\frac{1}{2^{n+1}}\right)=\{0\}$, $P_{T y}(1)=\{1\}$, and $\left(\frac{1}{2^{n+1}}, 0\right),(1,1) \in E(G)$.

If $(x, y)=(0,0)$ then $T x=\{0,1\}=T y$. We have $P_{T y}(0)=\{0\}, P_{T y}(1)=\{1\}$, and $(0,0),(1,1) \in$ $E(G)$.

If $(x, y)=\left(\frac{1}{2^{n}}, \frac{1}{2^{n}}\right)$, then $T x=T y=\left\{\frac{1}{2^{n+1}}, 1\right\}$ which obviously implies that $P_{T y}\left(\frac{1}{2^{n+1}}\right)=\left\{\frac{1}{2^{n+1}}\right\}$, $P_{T y}(1)=\{1\}$ and $\left(\frac{1}{2^{n+1}}, \frac{1}{2^{n+1}}\right),(1,1) \in E(G)$. So, $T$ is weak graph preserving. Next, we can see that $\frac{1}{2^{n+1}} \in T\left(\frac{1}{2^{n}}\right)$ and $\left(\frac{1}{2^{n}}, \frac{1}{2^{n+1}}\right) \in E(G)$. So the condition (2) of Theorem 3.1 is satisfied. Also, it is obvious that the condition (3) of Theorem 3.1 is satisfied. Thus all conditions of Theorem 3.1 are obtained. Therefore we can conclude that $T$ has a fixed point and the fixed point set $\operatorname{Fix}(T)=\{0\}$.

(2) $T$ is not graph preserving since $\frac{1}{2} \in T 1,1 \in T 0$ but $\left(\frac{1}{2}, 1\right) \notin E(G)$.

(3) $T$ is not a multi-valued contraction in the sense of Nicolae, O'Regan, and Petrusel since $(1,0) \in E(G), \frac{1}{2} \in T 1,1 \in T 0$ and $d\left(\frac{1}{2}, 1\right)<\alpha d(1,0)$ with $\alpha>\frac{1}{2}$ but $\left(\frac{1}{2}, 1\right) \notin E(G)$.

Remark 3.8 As a consequence of Example 3.7, we can neither use Theorem 1.13 nor Theorem 1.8 to check whether or not $T$ has a fixed point. 


\section{Acknowledgements}

This paper was supported by the Faculty of Science and Technology, Prince of Songkla University, Pattani Campus.

\section{Received: 20 July 2014 Accepted: 8 December 2014 Published: 18 Dec 2014}

\section{References}

1. Bojor, F: Fixed point theorems for Reich type contraction on metric spaces with a graph. Nonlinear Anal. 75, 3895-3901 (2012)

2. Branciari, A: A fixed point theorem for mappings satisfying a general contractive condition of integral type. J. Math. Anal. Appl. 29, 531-536 (2002)

3. Browder, FE: The fixed point theory of multi-valued mapping in topological spaces. Math. Ann. 177, 283-301 (1968)

4. Du, WS: New cone fixed point theorems for nonlinear multi-valued maps with applications. Appl. Math. Lett. 24(2), 172-178 (2011)

5. Feng, Y, Liu, S: Fixed point theorems for multi-valued contractive mappings and multi-valued Caristi type mappings. J. Math. Anal. Appl. 317, 103-112 (2006)

6. Khaojasten, F, Abbas, M, Costache, S: Two new types of fixed point theorems in complete metric spaces. Abstr. Appl. Anal. 2014, Article ID 325840 (2014)

7. Laza, T, Mot, G, Petrusel, G, Szentesi, S: The theory of Reich's fixed point theorem for multi-valued operators. Fixed Point Theory Appl. (2010). doi:10.1155/2010/178421

8. Minak, G, Acar, O, Altun, I: Multi-valued pseudo-Picard operators and fixed point results. J. Funct. Spaces Appl. 2013 Article ID 827458 (2013)

9. Petrusel, A, Petrusel, G: Selection theorems for multi-valued generalized contractions. Math. Morav. 9, 43-52 (2005)

10. Sitthithakerngkiet, $K$, Plubtieng, $S$ : Existence solutions of vector equilibrium problems and fixed point of multi-valued mappings. Abstr. Appl. Anal. 2013, Article ID 952021 (2013)

11. Ume, JS, Lee, BS, Cho, SJ: Some results on fixed point theorems for multi-valued mappings in complete metric spaces. Int. J. Math. Math. Sci. 30(6), 319-325 (2002)

12. Nadler, SB: Multi-valued contraction mappings. Pac. J. Math. 30, 475-488 (1969)

13. Kirk, WA, Massa, S: Remarks on asymptotic and Chebyshev centers. Houst. J. Math. 16, $465-468$ (1990)

14. Reich, S: Fixed points of contractive functions. Boll. Unione Mat. Ital. 5, 26-42 (1972)

15. Mizoguchi, N, Takahashi, W: Fixed point theorems for multi-valued mapping on complete metric spaces. J. Math. Anal. Appl. 141, 177-188 (1989)

16. Berinde, M, Berinde, V: On a general class multi-valued weakly Picard mappings. J. Math. Anal. Appl. 326, 772-782 (2007)

17. Jachymski, J: Fixed the contraction principle for mappings on a metric with a graph. Proc. Am. Math. Soc. 139, 1359-1373 (2008)

18. Beg, I, Butt, AR: Fixed point for set valued mappings satisfying an implicit relation in partially ordered metric spaces Nonlinear Anal. 71, 3699-3704 (2009)

19. Nicolae, A, O'Regan, D, Petrusel, A: Fixed point theorems for single-valued and multi-valued generalized contractions in metric spaces endowed with a graph. Georgian Math. J. 18, 307-327 (2011)

20. Dinevari, T, Frigon, T: Fixed point results for multi-valued contractions on a metric space with a graph. J. Math. Anal. Appl. 405, 507-517 (2013)

21. Tiammee, J, Suantai, S: Coincidence point theorems for graph-preserving multi-valued mappings. Fixed Point Theory Appl. (2014). doi:10.1186/1687-1812-2014-70

10.1186/1687-1812-2014-248

Cite this article as: Phon-on et al.: Coincidence point theorems for weak graph preserving multi-valued mapping. Fixed Point Theory and Applications 2014, 2014:248

\section{Submit your manuscript to a SpringerOpen ${ }^{\circ}$ journal and benefit from:}

- Convenient online submission

Rigorous peer review

- Immediate publication on acceptance

- Open access: articles freely available online

- High visibility within the field

- Retaining the copyright to your article 